\title{
Effect of gemigliptin on cardiac ischemia/reperfusion and spontaneous hypertensive rat models
}

\author{
Dae-Hwan Nam ${ }^{1,2}$, Jinsook Park ${ }^{3}$, Sun-Hyun Park ${ }^{1}$, Ki-Suk Kim ${ }^{1,4, *}$, and Eun Bok Baek ${ }^{3, *}$ \\ 'Predictive Model Research Center, Korea Institute of Toxicology, Daejeon 34114, Korea, ${ }^{2}$ Center for Inflammation, Immunity \& Infection, Institute for \\ Biomedical Sciences, Georgia State University, Atlanta, GA 30303, USA, ${ }^{3}$ Corporate R\&D, LG Chem, Ltd., Daejeon 34122, ${ }^{4}$ Department of Human and \\ Environmental Toxicology, University of Science and Technology, Daejeon 34113, Korea
}

\section{ARTICLE INFO}

Received April 16, 2019

Revised July 23, 2019

Accepted July 24, 2019

\section{*Correspondence \\ Ki-Suk Kim \\ E-mail: idkks@kitox.re.kr \\ Eun Bok Baek \\ E-mail: baekeunbok@hanmail.net}

\section{Key Words}

Dipeptidyl peptidase-IV

Gemigliptin

Myocardial ischemia-reperfusion injury

Pressure-volume loop

Spontaneously hypertensive rat

\begin{abstract}
Diabetes is associated with an increased risk of cardiovascular complications. Dipeptidyl peptidase-4 (DPP-IV) inhibitors are used clinically to reduce high blood glucose levels as an antidiabetic agent. However, the effect of the DPP-IV inhibitor gemigliptin on ischemia/reperfusion (I/R)-induced myocardial injury and hypertension is unknown. In this study, we assessed the effects and mechanisms of gemigliptin in rat models of myocardial I/R injury and spontaneous hypertension. Gemigliptin (20 and $100 \mathrm{mg} / \mathrm{kg} / \mathrm{d}$ ) or vehicle was administered intragastrically to Sprague-Dawley rats for 4 weeks before induction of I/R injury. Gemigliptin exerted a preventive effect on I/R injury by improving hemodynamic function and reducing infarct size compared to the vehicle control group. Moreover, administration of gemigliptin $(0.03 \%$ and $0.15 \%)$ powder in food for 4 weeks reversed hypertrophy and improved diastolic function in spontaneously hypertensive rats. We report here a novel effect of the gemigliptin on I/R injury and hypertension.
\end{abstract}

\section{INTRODUCTION}

Diabetes mellitus is an independent risk factor for cardiovascular $(\mathrm{CV})$ complications and mortality following myocardial infarction [1,2]. Diabetes mellitus also increases the risk of $\mathrm{CV}$ disease (CVD) [3], which is responsible for the rising incidence of coronary artery disease and stroke, and the increasing risk of heart failure [4].

Type 2 diabetes can be treated with biguanides (such as metformin), thiazolidinediones (such as pioglitazone and rosiglitazone), sulfonylurea, inhibitors of sodium-glucose cotransporter 2 and dipeptidyl peptidase-4 (DPP-IV) inhibitors, also known as gliptins [5]. DPP-IV inhibitors prevent degradation of glucagonlike peptide-1 (GLP-1) by DPP-IV [6], a cell-surface protease that selectively cleaves the N-terminal dipeptide from peptides with proline or alanine position [7]. Therefore, inhibitors of DPP-IV can show various physiological effects via incretin hormones, neuropeptides and other physiologically important peptides and proteins. Many studies have reported that marketed DPP-IV inhibitors have a protective effect on the cardiovascular system beyond their glucose lowering effect [8]. The DPP-IV inhibitors sitagliptin, linagliptin, alogliptin, and saxagliptin have been approved by the United States Food and Drug Administration and reportedly exert cardioprotective effects [9-12] via GLP1-dependent and -independent mechanisms. Binding of GLP-1 to its receptor (GLP-1R) results in the induction of vasodilation by promoting the release of nitric oxide from vascular endothelial cells via the cyclic adenosine monophosphate/protein kinase A pathway. Other substrates of DPP-IV, such as stromal cell-derived factor- $1 \alpha$, substance $\mathrm{P}$, and brain natriuretic peptide, may also have beneficial effects on the CV system [13].

Gemigliptin is a DPP-IV inhibitor that has been approved in

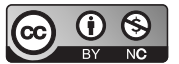

This is an Open Access article distributed under the terms of the Creative Commons Attribution Non-Commercial License, which permits unrestricted non-commercial use, distribution, and reproduction in any medium, provided the original work is properly cited. Copyright @ Korean J Physiol Pharmacol, pISSN 1226-4512, elSSN 2093-3827
Author contributions: D.H.N. conceived and designed the experiments. D.H.N., J.P., and S.H.P. performed there experiments. D.H.N. and E.B.B. wrote the original manuscript. S.H.P., K.S.K., and E.B.B. reviewed and edited the manuscript. E.B.B. and K.S.K. supervised and coordinated the study. 
the Republic of Korea and the global market. However, the effects of gemigliptin on animal models of CVD have not been reported. We assessed the effects of gemigliptin on rats with ischemia/reperfusion (I/R) injury and spontaneous hypertension.

\section{METHODS}

\section{Materials}

Gemigliptin tartrate sesquihydrate (LG Chemical, Daejeon, Korea), 2,3,5-triphenyl tetrazolium chloride (TTC; SigmaAldrich, St. Louis, MO, USA), isoflurane (Primal Healthcare, Andhra Pradesh, India), and Rompun (xylazine; Bayer, Leverkusen, Germany) were used in this study.

\section{Animals}

Male Sprague-Dawley ( $\mathrm{n}=7-9 ; 4$ weeks old; $180-200 \mathrm{~g}$ ), male Wistar-Kyoto (WKY, $\mathrm{n}=6$; 8 weeks old; 270-310 g) rat and male spontaneously hypertensive rats (SHR, $\mathrm{n}=8 ; 8$ weeks old; 270-310 g) were obtained from a commercial breeder (Orient Bio
Inc., Seongnam, Korea). All animals were housed with ad libitum access to water and food under a 12/12 h light/dark cycle at $22 \pm 2^{\circ} \mathrm{C}$. The rats were allowed to acclimatize for one week prior to the experiment. All procedures were approved by the Institutional Animal Care and Use Committee (IACUC) of the Korea Institute of Toxicology (IACUC approval No. 1704-0137).

\section{Surgical preparation}

I/R injury was induced in the rats by ligation of the left anterior descending (LAD) coronary artery for $10 \mathrm{~min}$ followed by reperfusion for $24 \mathrm{~h}$ [14]. Briefly, anesthesia was induced using a mixture of isoflurane $(3 \mathrm{ml} / \mathrm{min})$ and $95 \% \mathrm{O}_{2}$ and $5 \% \mathrm{CO}_{2}$ in an induction chamber. Rats were then sedated by intraperitoneal injection of Rompun $(1 \mathrm{mg} / \mathrm{kg})$. The trachea was intubated with a cannula connected to a Harvard rodent ventilator (Model 683; Harvard Apparatus, Holliston, MA, USA) for artificial respiration. The heart was exposed by performing a left thoracotomy, and the LAD was ligated 2-3 mm from its origin and loosened. During the period of ischemia, the body temperature of the rats was maintained by placing them on a $37^{\circ} \mathrm{C}$ heating pad (L.M.S. Korea, Seongnam, Korea). The rats were randomly divided ( $\mathrm{n}=$
A

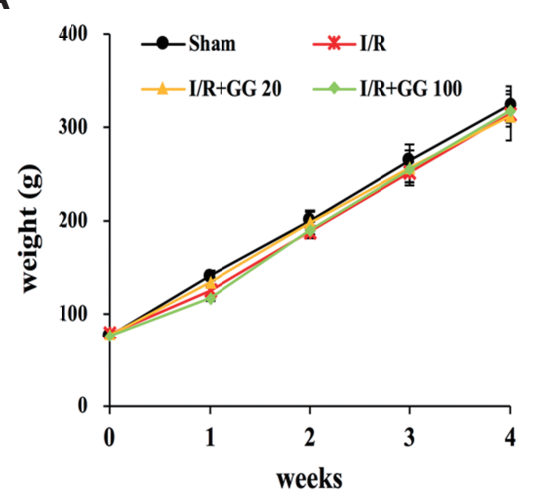

B

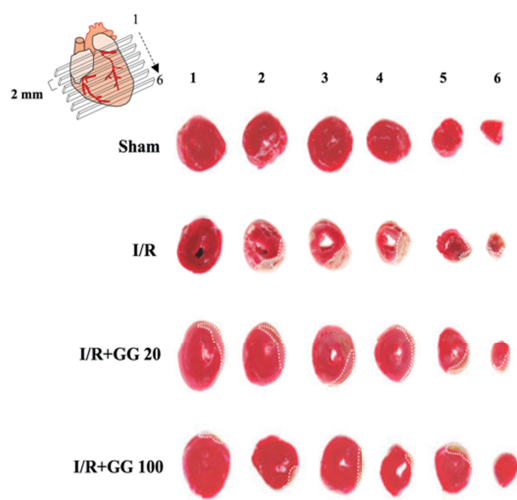

C

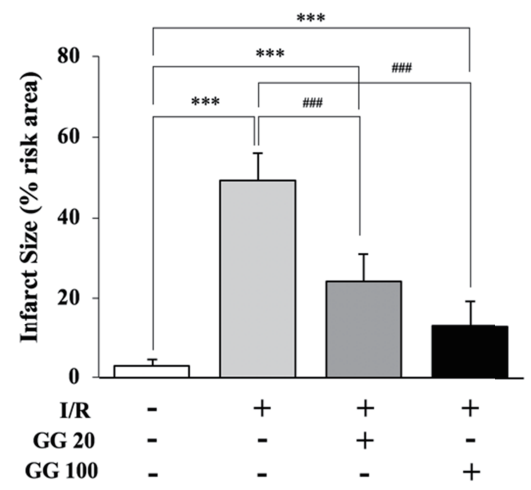

Fig. 1. Effect of gemigliptin (GG) on ischemia/reperfusion (I/R)-induced infarct size. (A) Change in rat weight as a function of the period of treatment. (B) Representative illustrations of heart sections stained with 2,3,5-triphenyl tetrazolium chloride (TTC). The infarcted area is shown in white color. The white dotted lines indicate the infarcted areas. Six sections cut into $2 \mathrm{~mm}$ transverse of the heart to visualize the infarcted areas. (C) Infarct size is quantified as a percentage of total slice area. All data are shown as mean \pm standard error of the mean. $\mathrm{N}=$ 7-9 hearts per group, Sham vs. I/R, I/R+GG $20, \mathrm{l} / \mathrm{R}+\mathrm{GG} 100\left({ }^{* *} \mathrm{p}<0.01 ; * * * \mathrm{p}<0.001\right)$, I/R vs. I/R+GG 20, I/R+GG $100\left({ }^{\# \#} \mathrm{p}<0.01\right.$; $\# \mathrm{p}<0.001)$. 
7-9) into the (1) sham group (underwent surgery but not I/R); (2) $\mathrm{I} / \mathrm{R}$ group (induction of $\mathrm{I} / \mathrm{R}$ ); and $(3,4)$ gemigliptin $+\mathrm{I} / \mathrm{R}$ groups (administered 20 or $100 \mathrm{mg} / \mathrm{kg}$ gemigliptin in distilled water intragastrically for 4 weeks before induction of $\mathrm{I} / \mathrm{R}$ ).

\section{Hypertensive rat model and treatment regimen}

Male WKY and SHR rats were randomly divided into four equal-sized groups: (1) WKY group, normotensive WKY rats; (2) hypertension group, SHR rats; and $(3,4)$ gemigliptin + hypertension groups, SHR rats fed gemigliptin powder $(0.03 \%$ and $0.15 \%)$ for four weeks.

\section{Drug administration}

Gemigliptin tartrate sesquihydrate powder was suspended in distilled water and administered daily to rats at 20 or $100 \mathrm{mg} /$ $\mathrm{kg}$ of body weight by oral gavage. Also, gemigliptin powder was mixed into the rats food in the gemigliptin + hypertension groups.

\section{Hemodynamic measurements}

Left ventricle (LV) function was evaluated using a pressurevolume (P-V) conductance catheter (ADV500 Admittance Pressure Volume Control Unit; Transonic Scisense Inc., London, ON, Canada). The catheter (1.9 F; Transonic Scisense Inc.) was inserted into the right carotid artery and advanced into the LV chamber to record the LV P-V relationship. We evaluated the following hemodynamic parameters: heart rate (HR), end systolic pressure (ESP), end diastolic pressure (EDP), $\mathrm{dP} / \mathrm{dt}$ maximum ( $\mathrm{dP} /$ $\mathrm{dt}$ max), dP/dt minimum (dP/dt min), end systolic volume (ESV), end diastolic volume (EDV), stroke volume (SV), cardiac output (CO), ejection fraction (EF), and stroke work (SW).

\section{Myocardial infarct analysis}

TTC (Sigma-Aldrich) staining was performed to evaluate myocardial infarct size. After reperfusion, the hearts were isolated, washed in phosphate-buffered saline (PBS) and sectioned into $2 \mathrm{~mm}$ transverse slices. After incubation in $1 \%$ TTC at $37^{\circ} \mathrm{C}$ in PBS for $15 \mathrm{~min}$, the heart slices were imaged. Ischemic areas (red) and infarct areas (white) were determined. The ratio of infarcted myocardium to total myocardial tissue was calculated using the infarct area / total heart area (\%). Infarct sizes were measured using Image J software (National Institutes of Health, Bethesda, MD, USA).

\section{Statistical analysis}

Results are expressed as means \pm standard error of the mean. Data were subjected to one-way analysis of variance with Tukey's post-hoc test using Prism 5.01 (GraphPad Software Inc., La Jolla, CA, USA). Values of $p<0.05,0.01$ and 0.01 were considered to indicate significant and highly significant differences, respectively.

\section{RESULTS}

\section{Gemigliptin reduces myocardial damage following I/R injury}

Body weight did not differ significantly among the four groups at days 1, 2, 3 and 4 (Fig. 1A). Therefore, it seemed gemigliptin was not toxic to the rats.

To examine the cardioprotective effects of gemigliptin, the size of the infarcted myocardium was determined through TTC staining. TTC stains healthy viable myocardium deep-red color, while pale white color as necrosis myocardium. Myocardial in-
A
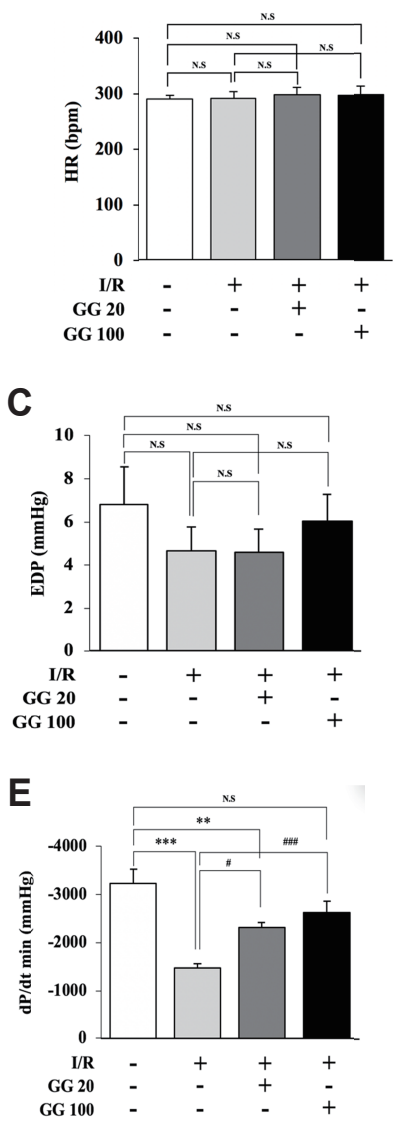

B

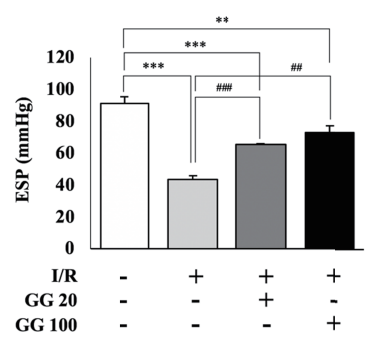

D

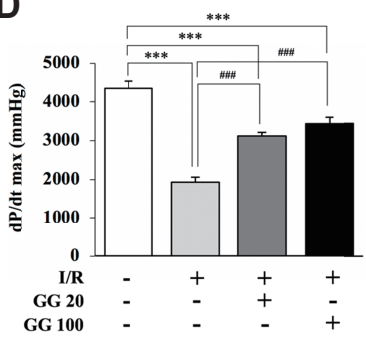

Fig. 2. Effects of gemigliptin (GG) on heart rate and mean blood pressure after ischemia/reperfusion (I/R)-induced injury. (A) $H R$, heart rate (beats/min); (B) ESP, end-systolic pressure $(\mathrm{mmHg})$; (C) EDP, end-diastolic pressure $(\mathrm{mmHg})$; and $(\mathrm{D}$ and $\mathrm{E})$ maximum and minimum $\mathrm{dP} / \mathrm{dt}$. All data are shown as mean \pm standard error of the mean. $\mathrm{N}=7-9$ hearts per group, Sham vs. I/R, I/R+GG 20 (GG $20 \mathrm{mg} / \mathrm{kg}$ ), and I/R+GG $100(\mathrm{GG} 100 \mathrm{mg} / \mathrm{kg})$ rats $\left({ }^{* *} \mathrm{p}<0.01 ;{ }^{* * *} \mathrm{p}<0.001\right)$; I/R vs. I/R+GG 20 and I/R+GG $100\left({ }^{\#} \mathrm{p}<0.05 ;{ }^{\# \#} \mathrm{p}<0.01 ;{ }^{\# \# \#} \mathrm{p}<0.001\right)$. NS, not significant. 
farcts in the $\mathrm{I} / \mathrm{R}$ group were significantly larger than those in the sham group and were reduced by treatment with 20 and $100 \mathrm{mg} /$ kg gemigliptin (Fig. 1B, C). These results demonstrated that gemigliptin had a protective effect against I/R-induced myocardial damage.

\section{Gemigliptin improves the recovery of cardiac blood pressure}

HR and EDP did not differ among the groups (Fig. 2A, C). Compared with the I/R group, the I/R + gemigliptin 20 and 100 $\mathrm{mg} / \mathrm{kg}$ groups showed significantly elevated ESP, dP/dt max, and $\mathrm{dP} / \mathrm{dt}$ min values (Fig. 2B, D, E). These data suggested that gemigliptin improved against I/R-induced decline in cardiac contractile function at the dosages tested.

\section{Gemigliptin improves functional recovery following I/R injury}

We next investigated the functional mechanism underlying the protective effect of gemigliptin on I/Rinduced cardiomyocyte injury. The ESV and EDV were significantly higher while the $\mathrm{SV}, \mathrm{CO}, \mathrm{EF}$, and SW were significantly lower in the I/R group compared with the sham group. However, all these values were improved by gemigliptin (Fig. 3A-F). The I/R group had a significantly lower systolic LV pressure than that of the gemigliptin groups (Fig. 3G). Furthermore, the loops of rats in the I/R group showed a prominent rightward displacement along the LV volume axis, suggesting cardiac injury. Therefore, gemigliptin increased the LV contraction and relaxation rates after I/R.

\section{Effect of gemigliptin on CV parameters on SHR model}

$\mathrm{I} / \mathrm{R}$ injury and hypertension induce LV remodeling and alter LV function. Gemigliptin exerted significant effects on the ESP, $\mathrm{dP} / \mathrm{dt}$ max, $\mathrm{dP} / \mathrm{dt}$ min, ESV, and EF values, but not on HR (Fig. 4A-F). The slope of the end-diastolic P-V relationship was steeper in SHRs than WKY rats (Fig. 4G) and was decreased by gemigliptin. Therefore, gemigliptin reduced LV hypertrophy and improved LV compliance.

\section{DISCUSSION}

In the present study, we report here for the first time that gemigliptin exerts a protective and preventive effect on $\mathrm{I} / \mathrm{R}$ injury and hypertension in a dose-dependent manner, respectively. We show that gemigliptin prevented I/R injury by improving the $\mathrm{EF}, \mathrm{dP} / \mathrm{dt}$ min, $\mathrm{dP} / \mathrm{dt}$ max, ESP, and infarct size and decreasing the ESV and EDV, but did not affect HR. Also, gemigliptin treatment in SHRs showed a significant reduction in blood pressure without changes in heart rate. Therefore, long-term administration of gemigliptin may exert a cardioprotective and preventive effect.

Gemigliptin is an oral diabetic agent for the treatment of type 2 diabetes by inhibiting DPP-IV [15]. Administration of 20 or
A

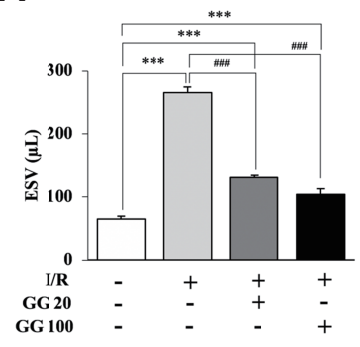

C

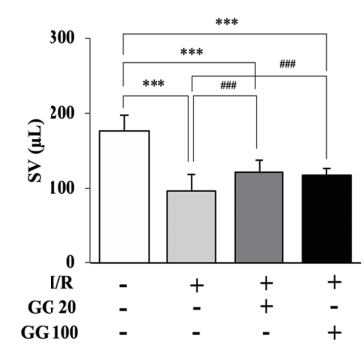

E

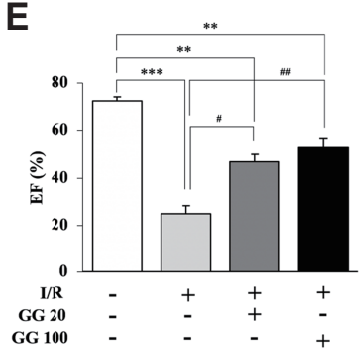

B

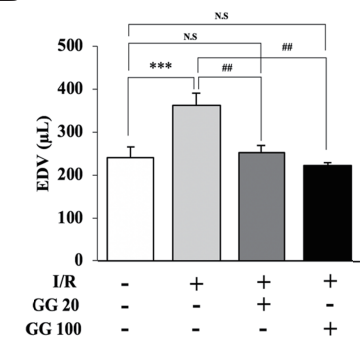

D

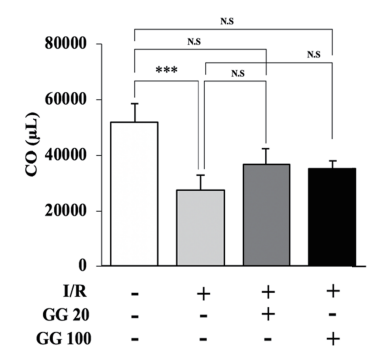

$\mathbf{F}$

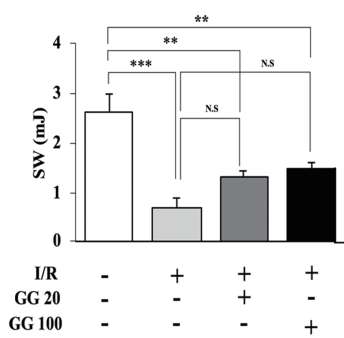

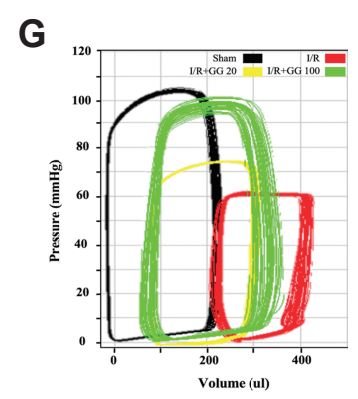

Fig. 3. Effects of gemigliptin (GG) on left ventricular function during ischemia/reperfusion (I/R)-induced injury. (A) ESV, end-systolic volume $(\mu \mathrm{l})$; (B) EDV, end-diastolic volume ( $\mu \mathrm{l})$; (C) SV, stroke volume ( $\mu \mathrm{l})$; (D) CO, cardiac output ( $\mu \mathrm{l})$; (E) EF, ejection fraction (\%); (F) SW, stroke work ( $\mathrm{mJ}$ ); and $(\mathrm{G})$ Representative pressure-volume loop values of sham, I/R, I/R+GG $20 \mathrm{mg} / \mathrm{kg}$, and I/R+GG $100 \mathrm{mg} / \mathrm{kg}$ rats. Sham (black), $\mathrm{I} / \mathrm{R}$ (red), I/R+GG $20 \mathrm{mg} / \mathrm{kg}$ (yellow), and I/R+GG $100 \mathrm{mg} / \mathrm{kg}$ (green). All data are shown as mean \pm standard error of the mean. $\mathrm{N}=7-9$ hearts per group, Sham vs. I/R, I/R+GG 20, and I/R+GG $100(* * p<0.01$; $\left.{ }^{* * *} \mathrm{p}<0.001\right)$; I/R vs. I/R+GG 20 and I/R+GG $100\left({ }^{*} \mathrm{p}<0.05 ;{ }^{* \#} \mathrm{p}<0.01\right.$; $\#$ \# $<0.001)$. NS, not significant. 
A

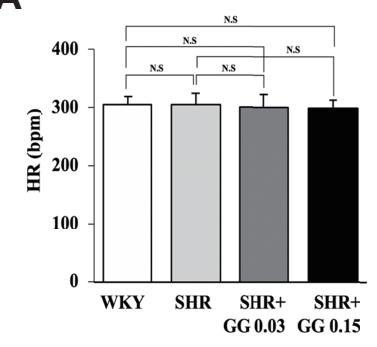

C

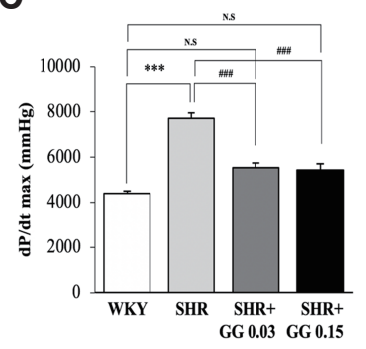

E

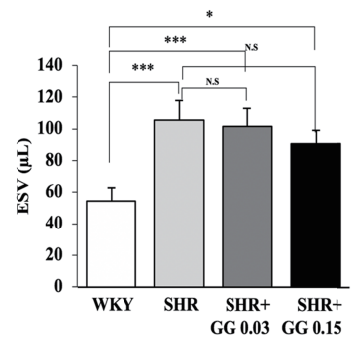

G

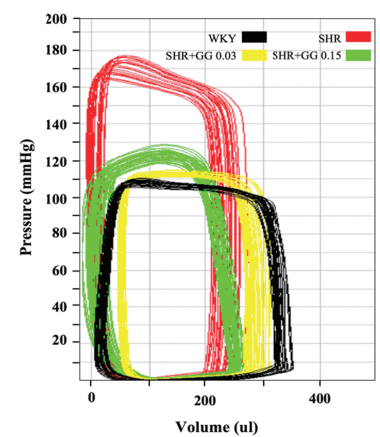

B

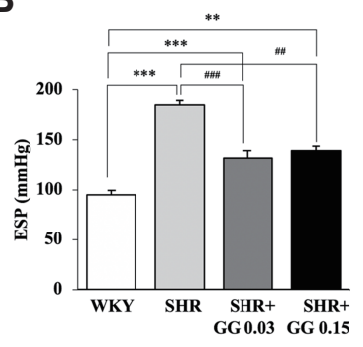

D

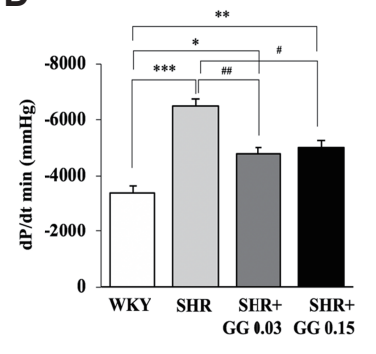

$\mathbf{F}$

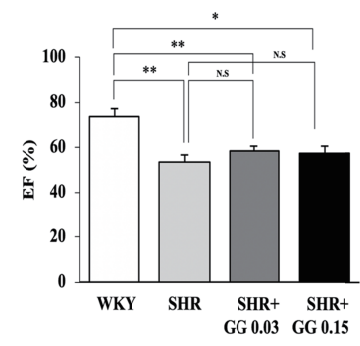

Fig. 4. Effects of gemigliptin (GG) on hemodynamic parameters in spontaneously hypertensive rats (SHR) model. (A) HR, heart rate (beats/min); (B) ESP, endsystolic pressure $(\mathrm{mmHg}) ;(C, D)$ maximum and minimum dP/dt; (E) ESV, end-systolic volume ( $\mu \mathrm{l}) ;(\mathrm{F}) \mathrm{EF}$, ejection fraction (\%); and (G) Representative pressure-volume loop values of Wistar-Kyoto (WKY), SHR, SHR+GG $0.03 \mathrm{mg} / \mathrm{kg}$, and SHR+GG $0.15 \mathrm{mg} / \mathrm{kg}$ rats. WKY (black), SHR (red), SHR+GG $0.03 \mathrm{mg} / \mathrm{kg}$ (yellow), and SHR+GG $0.15 \mathrm{mg} / \mathrm{kg}$ rats (green). All data are shown as mean \pm standard error of the mean. $\mathrm{N}=7-9$ hearts per group, WKY vs. SHR, SHR+GG 0.03, and SHR+GG $0.15\left({ }^{*} \mathrm{p}<0.05 ;{ }^{* *} \mathrm{p}<0.01 ; * * * \mathrm{p}<0.001\right)$; SHR vs. SHR+GG 0.03 and SHR+GG $0.15(" \mathrm{p}<0.05 ; \# \mathrm{p}<0.01 ; \# \mathrm{p}<0.001)$. NS, not significant.

$100 \mathrm{mg} / \mathrm{kg}$ of gemigliptin by oral gavage results in an increase in the GLP-1 level [16]. Following the administration of a single oral dose of gemigliptin ( 20 or $100 \mathrm{mg} / \mathrm{kg}$ ) to healthy subjects, its

mean plasma concentration was approximately 5- to 6-fold and 27- and 30-fold, respectively, of the clinical dose of $50 \mathrm{mg}$ [17]. In this study, gemigliptin increased GLP-1 activation in a dosedependent manner, and the plasma concentration was higher than the clinical dose used in humans (data not shown). Several mechanisms for the improvement of cardiac function by DPP-IV inhibitors have been suggested. Sitagliptin and vildagliptin exert a cardioprotective effect by increasing the expression of GLP-1 in a glucose-dependent manner [18]. Saxagliptin, via its antioxidant activity, exert a cardioprotective effect against I/R injury in diabetic and normoglycemic rats [19]. Other substrates of DPP-IV, such as chemokines, neuropeptides, and stromal cell-derived factor-1, may contribute to the protective effects of DPP-IV inhibitors against I/R injury [20].

The ESP was significantly higher in SH rats and was reduced by gemigliptin. We measured the plasma concentration of DPPIV after analyzing cardiac function, and gemigliptin-dosedependent effects on the concentrations of DPP-IV were observed between $0.03 \%$ and $0.15 \%$ of gemigliptin (data not shown). Gemigliptin $0.03 \%$ and $0.15 \%$ is equivalent to 20 and $100 \mathrm{mg} / \mathrm{kg} / \mathrm{day}$, respectively, based on daily food intake. However, plasma concentrations of DPP-IV were higher in the I/R rats than in the SH rats (data not shown). DPP-IV inhibitors reduce blood pressure mainly by improving endothelial function [21] and possibly by regulating cardiac calcium levels and glucose transporter 4 translocation [2123].

This study had several limitations. We did not determine the mechanism of the CV effects of gemigliptin; however, the CV effects were likely independent of its glucose-lowering effect because the models used did not involve high glucose levels. Further studies should examine the mechanisms underlying the abovementioned effects of gemigliptin.

In summary, gemigliptin exerted a cardioprotective and preventive effect by reducing the size of cardiac infarcts and lowering blood pressure. Therefore, gemigliptin exerts a beneficial effect on blood glucose and the CV system in the presence of cardiac I/ R injury.

\section{ACKNOWLEDGEMENTS}

We are thankful to the Corporate R\&D, LG Chem, Ltd for providing Gemigliptin tartrate sesquihydrate. We are also grateful to the Predictive Model Research Center, Korea Institute of Toxicology for helping us accomplish this research.

\section{CONFLICTS OF INTEREST}

The authors declare no conflicts of interest. 


\section{REFERENCES}

1. Daousi C, Casson IF, Gill GV, MacFarlane IA, Wilding JP, Pinkney $\mathrm{JH}$. Prevalence of obesity in type 2 diabetes in secondary care: association with cardiovascular risk factors. Postgrad Med J. 2006; 82:280-284.

2. Haffner SM, Lehto S, Rönnemaa T, Pyörälä K, Laakso M. Mortality from coronary heart disease in subjects with type 2 diabetes and in nondiabetic subjects with and without prior myocardial infarction. NEngl J Med. 1998;339:229-234.

3. Rao Kondapally Seshasai S, Kaptoge S, Thompson A, Di Angelantonio E, Gao P, Sarwar N, Whincup PH, Mukamal KJ, Gillum RF, Holme I, Njølstad I, Fletcher A, Nilsson P, Lewington S, Collins R, Gudnason V, Thompson SG, Sattar N, Selvin E, Hu FB, et al.; Emerging Risk Factors Collaboration. Diabetes mellitus, fasting glucose, and risk of cause-specific death. N Engl J Med. 2011;364:829841.

4. Martín-Timón I, Sevillano-Collantes C, Segura-Galindo A, Del Cañizo-Gómez FJ. Type 2 diabetes and cardiovascular disease: have all risk factors the same strength? World J Diabetes. 2014;5:444-470.

5. Dardano A, Penno G, Del Prato S, Miccoli R. Optimal therapy of type 2 diabetes: a controversial challenge. Aging (Albany NY). 2014;6:187-206.

6. Ahrén B. Dipeptidyl peptidase-4 inhibitors: clinical data and clinical implications. Diabetes Care. 2007;30:1344-1350.

7. Lambeir AM, Durinx C, Scharpé S, De Meester I. Dipeptidyl-peptidase IV from bench to bedside: an update on structural properties, functions, and clinical aspects of the enzyme DPP IV. Crit Rev Clin Lab Sci. 2003;40:209-294.

8. Fadini GP, Avogaro A. Cardiovascular effects of DPP-4 inhibition: beyond GLP-1. Vascul Pharmacol. 2011;55:10-16.

9. Sauvé M, Ban K, Momen MA, Zhou YQ, Henkelman RM, Husain $\mathrm{M}$, Drucker DJ. Genetic deletion or pharmacological inhibition of dipeptidyl peptidase- 4 improves cardiovascular outcomes after myocardial infarction in mice. Diabetes. 2010;59:1063-1073.

10. Esposito G, Cappetta D, Russo R, Rivellino A, Ciuffreda LP, Roviezzo F, Piegari E, Berrino L, Rossi F, De Angelis A, Urbanek K. Sitagliptin reduces inflammation, fibrosis and preserves diastolic function in a rat model of heart failure with preserved ejection fraction. Br J Pharmacol. 2017;174:4070-4086.

11. Koibuchi N, Hasegawa Y, Katayama T, Toyama K, Uekawa K, Sueta D, Kusaka H, Ma M, Nakagawa T, Lin B, Kim-Mitsuyama S. DPP-4 inhibitor linagliptin ameliorates cardiovascular injury in salt-sensitive hypertensive rats independently of blood glucose and blood pressure. Cardiovasc Diabetol. 2014;13:157.

12. Ihara $M$, Asanuma $H$, Yamazaki S, Kato H, Asano $Y$, Shinozaki $Y$, Mori H, Minamino T, Asakura M, Sugimachi M, Mochizuki N, Kitakaze M. An interaction between glucagon-like peptide-1 and adenosine contributes to cardioprotection of a dipeptidyl peptidase 4 inhibitor from myocardial ischemia-reperfusion injury. Am J Physiol Heart Circ Physiol. 2015;308:H1287-H1297.

13. Scheen AJ. GLP-1 receptor agonists and heart failure in diabetes. Diabetes Metab. 2017;43 Suppl 1:2S13-2S19.

14. Powers SK, Smuder AJ, Kavazis AN, Quindry JC. Mechanisms of exercise-induced cardioprotection. Physiology (Bethesda). 2014; 29:27-38.

15. Kim SH, Jung E, Yoon MK, Kwon OH, Hwang DM, Kim DW, Kim J, Lee SM, Yim HJ. Pharmacological profiles of gemigliptin (LC150444), a novel dipeptidyl peptidase-4 inhibitor, in vitro and in vivo. Eur J Pharmacol. 2016;788:54-64.

16. Kim SH, Yoo JH, Lee WJ, Park CY. Gemigliptin: an update of its clinical use in the management of type 2 diabetes mellitus. Diabetes Metab J. 2016;40:339-353.

17. Lim KS, Kim JR, Choi YJ, Shin KH, Kim KP, Hong JH, Cho JY, Shin HS, Yu KS, Shin SG, Kwon OH, Hwang DM, Kim JA, Jang IJ. Pharmacokinetics, pharmacodynamics, and tolerability of the dipeptidyl peptidase IV inhibitor LC15-0444 in healthy Korean men: a doseblock-randomized, double-blind, placebo-controlled, ascending single-dose, Phase I study. Clin Ther. 2008;30:1817-1830.

18. Hausenloy DJ, Whittington HJ, Wynne AM, Begum SS, Theodorou L, Riksen N, Mocanu MM, Yellon DM. Dipeptidyl peptidase-4 inhibitors and GLP-1 reduce myocardial infarct size in a glucosedependent manner. Cardiovasc Diabetol. 2013;12:154.

19. Mandlem VVK, Annapurna A. Cardioprotective role of saxagliptin through antioxidant mechanism in experimental myocardial infarction in STZ induced diabetic rats. J Clin Exp Pharmacol. 2017;7:1000233.

20. Ku HC, Chen WP, Su MJ. DPP4 deficiency preserves cardiac function via GLP-1 signaling in rats subjected to myocardial ischemia/ reperfusion. Naunyn Schmiedebergs Arch Pharmacol. 2011;384:197207.

21. Liu L, Liu J, Wong WT, Tian XY, Lau CW, Wang YX, Xu G, Pu Y, Zhu Z, Xu A, Lam KS, Chen ZY, Ng CF, Yao X, Huang Y. Dipeptidyl peptidase 4 inhibitor sitagliptin protects endothelial function in hypertension through a glucagon-like peptide 1-dependent mechanism. Hypertension. 2012;60:833-841.

22. Lee TI, Kao YH, Chen YC, Huang JH, Hsu MI, Chen YJ. The dipeptidyl peptidase- 4 inhibitor-sitagliptin modulates calcium dysregulation, inflammation, and PPARs in hypertensive cardiomyocytes. Int J Cardiol. 2013;168:5390-5395.

23. Giannocco G, Oliveira KC, Crajoinas RO, Venturini G, Salles TA, Fonseca-Alaniz MH, Maciel RM, Girardi AC. Dipeptidyl peptidase IV inhibition upregulates GLUT4 translocation and expression in heart and skeletal muscle of spontaneously hypertensive rats. Eur J Pharmacol. 2013;698:74-86. 